\title{
Diabetes insipidus in pregnancy
}

\author{
William M Hague MD FRCP FRCOG
}

Department of Obstetrics, University of Adelaide, Women's and Children's Hospital, North Adelaide SA 5006, Australia

\begin{abstract}
Summary: Diabetes insipidus is an uncommon condition with various aetiologies. Recent research has uncovered new mechanisms underlying the syndrome. Careful attention to management is essential in pregnant women to avoid serious complications. Diabetes insipidus in pregnancy may be due to relative reduction in secretion of AVP from the posterior pituitary (cranial DI), increase in breakdown of AVP by placental cystine aminopeptidase with vasopressinase activity, or resistance of the rental tubules to AVP (nephrogenic DI).
\end{abstract}

Keywords: diabetes insipidus, pregnancy, aquaporin, vasopressin, dDAVP

\section{PATHOPHYSIOLOGY OF DIABETES INSIPIDUS}

Diabetes insipidus (DI) is a condition where the kidney fails to concentrate its urinary output, and as a result the urine becomes increasingly dilute with water loss that can rapidly dehydrate the patient. The pathophysiology of DI relates to the effect of the posterior pituitary hormone arginine vasopressin $(\mathrm{AVP})$ on the renal tubules, controlling the efflux of water into the urine. Unlike diabetes mellitus, where the urine is sweet (mellitus - Greek for 'honeyed') because of its increased glucose concentration, the urine passed in DI is tasteless 'insipid': it is just water that is being passed. It was Marshall Lindheimer ${ }^{1}$ who coined the aphorism 'if the pregnant patient can't concentrate, the physician needs to', drawing attention to the problems facing the pregnant woman with DI. This article will provide some guidelines for those caring for such women.

The concentration of solute in a solvent has a direct effect on the passage of that solute across a semi-permeable membrane osmosis - and is measured as its osmolality. However, dilute urine has low osmolality, while haemocentration has high osmolality. In the normal mammalian fluid economy, osmotic equilibrium is maintained by the physiological osmostat, thought to be located in the anterior wall of the third ventricle: lesions in this area lead to a reduction in the response of AVP release to osmotic stimulation. ${ }^{2}$ There is also a non-osmotic contribution to AVP release from changes in blood volume, thought to be mediated through baro-receptors in the left atrium. $^{3}$ Animal experiments show that, provided blood volume is constant, osmotic changes produce a linear effect on AVP secretion. If, however, there is more than a $10 \%$ fall in blood volume, then there is an exponential increase in AVP secretion, overwhelming the osmotic regulation. ${ }^{4}$

Release of AVP blocks the transport of water from the blood across the tubular cells into the tubules, concentrating the urine and decreasing the concentration of solute, particularly sodium, in the blood until a homeostatic balance can be resolved by

Correspondence to: William M Hague

Email: bill.hague@adelaide.edu.au increased fluid intake. Conversely, fluid overload leads to a reduced secretion of AVP, allowing water loss into the tubules with increasingly dilute urine until the effect of the haemodilution is resolved, and the equilibrium is restored.

The effect of AVP is itself mediated by one or more of a family of recently recognized water channels, known as aquaporins (AQPs), whose discovery ${ }^{5}$ led to the award of a Nobel prize in 2003. These AQPs help to explain the differing transport of water across the various parts of the renal tubules and collecting ducts, and the associated counter current concentrating mechanism. ${ }^{6}$ The various members of the AQP family have different sensitivities to AVP. Thus, AQP1 is expressed in the proximal tubule and the descending limb of the loop of Henle, and is not under the control of AVP; absence of the gene leads to a reduction in the concentrating capacity of the kidney and associated polyuria. ${ }^{7}$ AQP2 and AQP3 are found in the collecting ducts and are both regulated by AVP: AQP3 knockout mice have polyuria that is unresponsive to AVP. ${ }^{8}$

In pregnancy, there is a change in the sensitivity of the osmostat, with reduced plasma sodium concentrations leading to a reduction in plasma osmolality, the mean falling from about 290 to $280 \mathrm{mosmol} / \mathrm{L}$ by $2-4$ weeks gestation in human pregnancy. ${ }^{9}$ There is also a reduction in the osmolar threshold for thirst. ${ }^{10}$ This reduction does not automatically lead to the usual response of cessation of AVP release, but its initiation may contribute to the frequency of micturition that is one of the hallmarks of the first trimester, even though the volume of urine passed is not different from that outside of pregnancy.

Human placenta produces a large amount of cystine aminopeptidase, which has both vasopressinase and oxytocinase activity, and whose increasing production as pregnancy proceeds parallels the increasing clearance rate of AVP, contributing a further balance to the change in water equilibrium in pregnancy. ${ }^{11}$

It has recently been shown in pregnant rats that there is a marked increase in the expression of AQP2 in the renal medulla, which can be blocked by administration of an AVP receptor antagonist. ${ }^{12}$ This receptor is also responsive to oxytocin, whose antidiuretic effect may also contribute to the water retention of pregnancy. 


\section{CLINICAL PRESENTATIONS OF DI}

DI occurs in various situations (Table 1). Either AVP fails to be released from the posterior pituitary, so-called 'central' DI, or AVP is metabolized before it can act on the tubules, or the tubules can become resistant to the effect of AVP, so-called 'nephrogenic' DI. The first is generally more common, and may relate to pituitary pathology, such as infarction of the pituitary because of a loss of blood supply, or an inflammatory process damaging the pituitary, or to a tumour compressing the pituitary. Increased metabolism of AVP may result from an increase in vasopressinase activity. Nephrogenic DI may be due to genetic mutations in AQP expression or production, or to mutations in the arginine vasopressin receptor 2 (AVPR2) gene that codes for the V2 receptor, $^{13}$ or to drug effects on the tubules, notably in patients taking lithium therapy. Exposure of a fetus to an angiotensin type 1 receptor blocker has also been reported to result in nephrogenic DI. Whatever the mechanism, the downstream effect is of increased water loss resulting in dilute urine and associated haemoconcentration.

DI may predate pregnancy, or may be unmasked by pregnancy because of the increased clearance of AVP by placental cystine aminopeptidase. It may also arise de novo during pregnancy and the puerperium as a complication of some other pathology.

The classical presentation of acute DI in relation to pregnancy is in Sheehan's syndrome, when severe haemorrhage in late pregnancy or after delivery leads to ischaemia or necrosis of the hypervascular pituitary, and is followed by polyuria and failure to lactate, associated with loss of posterior and anterior pituitary function, respectively, and later secondary hypoadrenalism and hypogonadism. ${ }^{14}$ The DI may be a late presentation of Sheehan's following on the administration of replacement glucocorticoids, as these are required by the distal renal tubules in the generation of free water. ${ }^{15}$

A less common cause of posterior pituitary failure in pregnancy is local pituitary or hypothalamic disease. A macroprolactinoma may enlarge during pregnancy, causing compression of the pituitary stalk and failure to release AVP, associated with bitemporal hemianopia. There may be incidental haemorrhage into the pituitary within a pre-existing tumour or cyst, usually presenting with severe headache and collapse. ${ }^{16}$

Table 1 Causes of diabetes insipidus (DI)

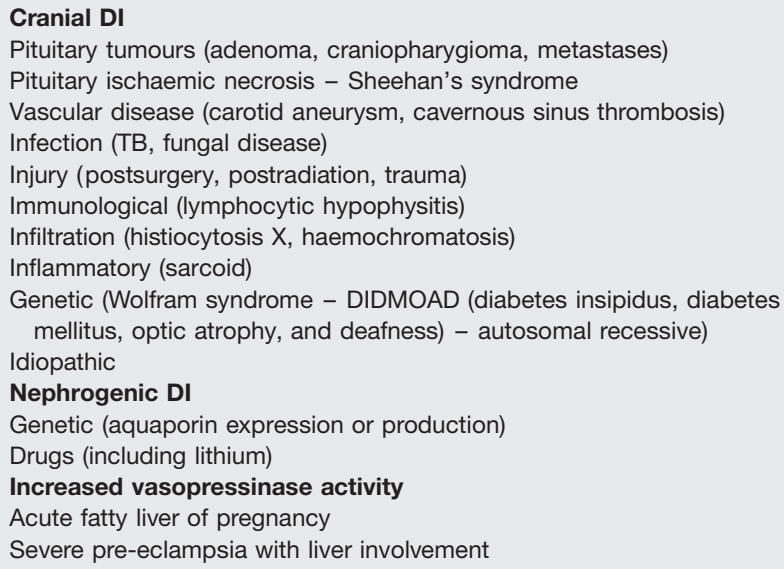

$\mathrm{TB}=$ tuberculosis
Lymphocytic hypophysitis is a rare autoimmune disease of the pituitary that typically presents in pregnancy or postpartum with acute or chronic hypopituitarism, including DI, in very much the same way as Sheehan's syndrome, but without a history of obstetric haemorrhage. Magnetic resonance imaging (MRI) displays a homogeneous symmetrical intrasellar mass with avid enhancement after administration of gadolinium, although cystic change has been described. The pituitary is replaced by a suprasellar mass of lymphocytes and plasma cells, and this has to be distinguished from a tumour by a biopsy. Pituitary antibodies have been found, but the sensitivity and specificity are low. The natural history of the disease is often of spontaneous resolution, with recovery of normal function, although there may be some long-term hormonal deficit. Some women with aggressive symptoms may require decompressive surgery. Treatment with high-dose steroids to reduce local inflammation is controversial. ${ }^{17}$ There are no randomized trials to guide treatment.

Transient DI of pregnancy is seen in relation to conditions where the metabolism of vasopressinase is altered, typically in disorders affecting the liver during pregnancy, such as acute fatty liver and severe pre-eclampsia with liver involvement (HELLP syndrome) ${ }^{18}$ The use of standard AVP in these conditions did not lead to the expected reduction in polyuria and associated hypernatraemia, whereas administration of its analogue, desmopressin (1-deamino-8-D-arginine vasopressin: dDAVP), which is more resistant to the effect of vasopressinase, proved to be generally effective. ${ }^{19}$ The DI resolves over the period of several weeks as the liver disease recovers, and does not usually recur in subsequent pregnancies.

A transient form of DI may also occur during pregnancy in the absence of liver disease. This usually represents an unveiling of subclinical cranial DI, with the posterior pituitary unable to respond to the physiological demands of pregnancy. ${ }^{20}$ In such cases, MRI shows loss of normal high signal intensity in the posterior pituitary in the TI-weighted images, consistent with loss of AVP secretion (Figure 1).

\section{ASSESSMENT AND MANAGEMENT OF DI IN PREGNANCY}

Treatment of central DI in pregnancy is to continue with, or to commence replacement of AVP with, dDAVP, coupled with fluid restriction of $1000-1500 \mathrm{~mL} /$ day. The dDAVP can be administered either intravenously or subcutaneously to initiate treatment $(2-5 \mu \mathrm{g})$, or as a tablet preparation or a nasal spray, which allows for fine tuning of the dose. The daily dose (usually around $20 \mu \mathrm{g}$ ) is commonly divided into two, with a bigger dose in the morning to mimic the usual diurnal variation of water balance. Care should be taken in women who develop renal insufficiency, e.g. in pre-eclampsia, when the clearance of $\mathrm{dDAVP}$ is reduced. The limited data available suggest that the use of dDAVP in pregnancy is safe. ${ }^{21}$

It should be remembered that, once administered, the effects of dDAVP cannot be reversed except by the passage of time, with mainly renal clearance and also some endogenous metabolism: there is no known antidote. Simultaneous administration of fluids, whether oral or parenteral, must be managed with great caution, because of the risk of inducing fluid overload and hyponatraemia, which can have disastrous consequences. Symptoms associated with hyponatraemia include headache, nausea and vomiting, weight gain, restlessness, fatigue, lethargy, disorientation, depressed reflexes, loss of appetite, 


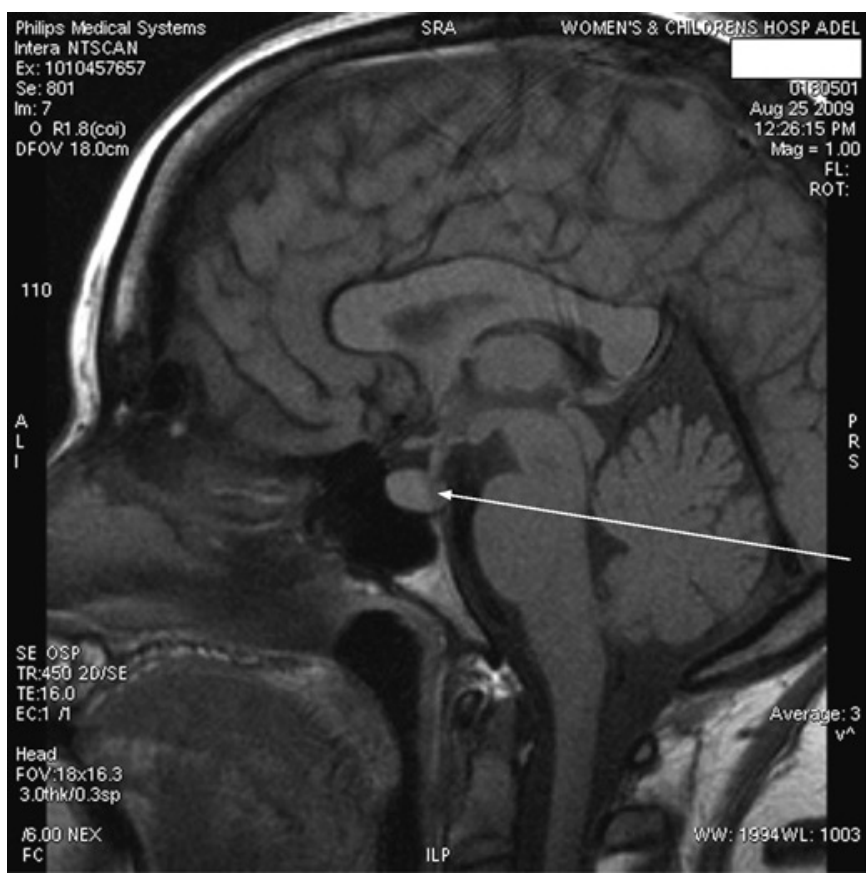

Figure $1 \mathrm{MRI}$ of a 35-year-old woman with de novo central diabetes insipidus at 20 weeks gestation in her third ongoing pregnancy, her first pregnancies having been normal, showing the typical features of loss of normal high signal intensity in the posterior pituitary in the T1-weighted images (arrow), consistent with loss of AVP secretion, with no other pathology identified. Diagnosis: transient gestational diabetes insipidus. AVP = arginine vasopressin; $\mathrm{MRI}=$ magnetic resonance imaging

irritability, muscle weakness, muscle spasms or cramps, and abnormal mental status, such as hallucinations, decreased consciousness and confusion. In severe cases, seizures and/or coma may occur. Overdose of dDAVP can also lead to oliguria and fluid retention. Depending on the clinical circumstances, the dose of dDAVP should be reduced, the frequency of administration lessened or the drug withdrawn.

The concomitant administration of drugs that may increase the risk of water intoxication with hyponatraemia (including tricyclic antidepressants, selective serotonin re-uptake inhibitors, phenothiazines, opiates, non-steroidal anti-inflammatory drugs (NSAIDs), lamotrigine and carbemazepine) should be undertaken with caution in women being treated with dDAVP.

The treatment of nephrogenic DI is more problematic in pregnancy. Water restriction is the mainstay of treatment. ${ }^{22}$ Drugs that have been used in this condition include thiazide diuretics and NSAIDs, whose use must be balanced against the potential risks for the gravid woman. In general, thiazides are not commonly used in pregnancy, because of the risk of reducing plasma volume. This may contribute to gestational syncope, but it may be particularly a problem in situations where there is utero-placental insufficiency, such as pre-eclampsia and intrauterine growth restriction. Other associated risks include electrolyte imbalance and neonatal thrombocytopenia. NSAIDs should be used with caution in the first trimester of pregnancy, because of an increased risk of miscarriage, and in the third trimester, because of the risk of closure of the fetal ductus arteriosus as well as of oligohydramnios and fetal renal impairment. There may be a place for their careful use in the second trimester of pregnancy.

Pregnant women presenting with thirst and polyuria in pregnancy in the absence of a known history of DI need careful assessment in hospital. Measurement of serum electrolytes, looking in particular for hypernatraemia, and of urine and serum osmolality, looking for inappropriately dilute urine in the face of concentrated serum, should be first line investigations, together with measurement of renal and liver function tests. Water deprivation tests may be performed, after an overnight fast where symptoms are mild, or in the morning if there is severe polyuria, watching carefully for weight loss as well as checking serial urine and serum osmolalities. Where liver transaminases are abnormal de novo, or there is renal impairment or hyperuricaemia, such women should be admitted to an obstetric High Dependency Unit or equivalent for work-up of possible acute fatty liver of pregnancy or severe pre-eclampsia with hepatic involvement. Pituitary imaging with MRI may be considered, in the absence of other obstetric pathology, to identify other local aetiologies for the DI. Close attention to fluid balance should be observed. Fluid restriction and careful initiation of dDAVP (as above) should be undertaken. If the patient is hypernatraemic at presentation, serum sodium concentrations should be followed every 4-6 hours: administration of dDAVP should be sufficient to halt the water loss and resolve the electrolyte imbalance. Collaboration with endocrinologists, who have expertise in the management of DI, is advised. Following delivery, there should be ongoing close attention to fluid balance, and daily assessment of the requirements for dDAVP, which may rapidly decrease, although more commonly there is a more gradual fall.

\section{DISCLOSURES}

The author has no conflicts of interest to declare.

\section{REFERENCES}

1 Lindheimer MD. Polyuria and pregnancy: its cause, its danger. Obstet Gynecol 2005;105(Part 2):1171-2

2 Thrasher TN. Osmoreceptor mediation of thirst and vasopressin secretion in the dog. Fed Proc 1982;41:2528-32

3 Gauer OH, Henry JP. Neurohormonal control of plasma volume. Int Rev Physiol 1976;9:145-90

4 Dunn FL, Brennan TJ, Nelson AE, Robertson GL. The role of blood osmolality and volume in regulating vasopressin secretion in the rat. J Clin Invest 1973;52:3212-9

5 Agre P, King LS, Yasui M, et al. Aquaporin water channels - from atomic structure to clinical medicine. J Physiol 2002;542(Part 1):3-16

6 Schrier RW. Body water homeostasis: clinical disorders of urinary dilution and concentration. J Am Soc Nephrol 2006;17:1820-32

7 King LS, Choi M, Fernandez PC, Cartron JP, Agre P. Defective urinary-concentrating ability due to a complete deficiency of aquaporin-1. N Engl J Med 2001;345:175-9

8 Ma T, Song Y, Yang B, et al. Nephrogenic diabetes insipidus in mice lacking aquaporin-3 water channels. Proc Natl Acad Sci USA 2000;97:4386-91

9 Davison JM, Vallotton MB, Lindheimer MD. Plasma osmolality and urinary concentration and dilution during and after pregnancy: evidence that lateral recumbency inhibits maximal urinary concentrating ability. $\mathrm{Br}$ J Obstet Gynaecol 1981;88:472-9

10 Lindheimer MD, Davison JM. Osmoregulation, the secretion of arginine vasopressin and its metabolism during pregnancy. Eur J Endocrinol 1995;132:133-43

11 Davison JM, Sheills EA, Philips PR, Barron WM, Lindheimer MD. Metabolic clearance of vasopressin and an analogue resistant to vasopressinase in human pregnancy. Am J Physiol 1993;264(Part 2):F348-53

12 Ohara M, Martin PY, Xu DL, et al. Upregulation of aquaporin 2 water channel expression in pregnant rats. J Clin Invest 1998;101:1076-83

13 Cheong HI, Park HW, Ha IS, et al. Six novel mutations in the vasopressin V2 receptor gene causing nephrogenic diabetes insipidus. Nephron 1997;75:431-7

14 Lust K, McIntyre HD, Morton A. Sheehan's syndrome - acute presentation with hyponatraemia and headache. Aust N Z J Obstet Gynaecol 2001;41:348-51 
15 Schwartz AR, Leddy AL. Recognition of diabetes insipidus in postpartum hypopituitarism. Obstet Gynecol 1982;59:394-8

16 Molitch ME. Pituitary tumors and pregnancy. Growth Hormone IGF Res 2003; 13(Suppl. 1):S38-44

17 Molitch ME, Gillam MR. Lymphocytic hypophysitis. Hormone Research 2007;68(Suppl. 5):145-50

18 Kennedy S, Hall PM, Seymour AE, Hague WM. Transient diabetes insipidus and acute fatty liver of pregnancy. Br J Obstet Gynaecol 1994;101:387-91

19 Durr JA. Diabetes insipidus in pregnancy. Am J Kidney Dis 1987;9:276-83
20 Williams DJ, Metcalfe KA, Skingle L, Stock AI, Beedham T, Monson JP. Pathophysiology of transient cranial diabetes insipidus during pregnancy. Clin Endocrinol (Oxf) 1993;38:595-600

21 Ray JG. DDAVP use during pregnancy: an analysis of its safety for mother and child. Obstet Gynecol Surv 1998;53:450-5

22 Seckl JR, Dunger DB. Diabetes insipidus. Current treatment recommendations. Drugs 1992;44:216-24 